\title{
Synergetic design of enlarged surface area and pseudo-capacitance for fiber-shaped supercapacitor yarn
}

Le, Thi Suong ; Truong, Thuy Kieu ; Huynh, Van Ngoc; Bae, Joonho ; Suh, Dongseok

Published in:

Nano Energy

Link to article, DOI:

10.1016/j.nanoen.2019.104198

Publication date:

2020

Document Version

Peer reviewed version

Link back to DTU Orbit

Citation (APA):

Le, T. S., Truong, T. K., Huynh, V. N., Bae, J., \& Suh, D. (2020). Synergetic design of enlarged surface area and pseudo-capacitance for fiber-shaped supercapacitor yarn. Nano Energy, 67, [104198].

https://doi.org/10.1016/..nanoen.2019.104198

\section{General rights}

Copyright and moral rights for the publications made accessible in the public portal are retained by the authors and/or other copyright owners and it is a condition of accessing publications that users recognise and abide by the legal requirements associated with these rights.

- Users may download and print one copy of any publication from the public portal for the purpose of private study or research.

- You may not further distribute the material or use it for any profit-making activity or commercial gain

- You may freely distribute the URL identifying the publication in the public portal 
Full paper

\title{
Synergetic design of enlarged surface area and pseudo-capacitance for fiber-shaped supercapacitor yarn
}

\author{
Thi Suong Le ${ }^{\mathrm{a}, \mathrm{b}}$, Thuy Kieu Truong ${ }^{\mathrm{c}}$, Van Ngoc Huynh ${ }^{\mathrm{d}}$, Joonho Bae ${ }^{\text {e,*** }}$, Dongseok Suh ${ }^{\mathrm{a}, \mathrm{b}, *}$ \\ ${ }^{a}$ Department of Energy Science, Sungkyunkwan University, Suwon, 16419, Republic of Korea \\ ${ }^{\mathrm{b}}$ Center for Integrated Nanostructure Physics, Institute for Basics Science, Suwon, 16419, Republic of Korea \\ ${ }^{c}$ Institute of Physics at Ho Chi Minh City, Vietnam Academy of Science and Technology, Ho Chi Minh, 710116, Viet Nam \\ d Department of Micro- and Nanotechnology, Technical University of Denmark, DK-2800, Kgs. Lyngby, Denmark \\ e Department of Nano-Physics, Gachon University, Seongnam-si, 13120, Republic of Korea
}

\section{A R T I C L E I N F O}

\section{Keywords:}

Yarn/fiber supercapacitor electrode

Carbon nanotube yarn

Zinc oxide nanorod

Nickel-cobalt layered double hydroxide

Flexible symmetric supercapacitor yarn

\begin{abstract}
A B S T R A C T
In various wearable energy storage devices, the shape of fiber or yarn has many advantages owing to their compatibility with the environment in which they are deployed. We present a systematic approach to maximizing the capacitance of a supercapacitor yarn by significantly increasing the yarn's surface area by growing a high density of nanorods around the yarn, followed by coating the surface with a pseudo-capacitive material. The two-step strategy is implemented using a dry-spun carbon nanotube yarn-based electrode, which is surrounded by a zinc oxide nanorod forest that is coated by a pseudo-capacitive nickel-cobalt layered double hydroxide material. The flexible as-prepared electrode exhibits a maximum capacitance of $1065 \mathrm{mF} \mathrm{cm}^{-2}\left(1278 \mathrm{~F} \mathrm{~g}^{-1}\right)$ at a scan rate of $5 \mathrm{mV} \mathrm{s}^{-1}$ and an excellent capacitance retention of $60.5 \%$ over 7000 cycles at a current density of $30 \mathrm{~mA} \mathrm{~cm}^{-2}$. The outstanding performance of the composite yarn supercapacitor can be ascribed to the enhanced ion accessibility to the deep surface of the nickel-cobalt layered double hydroxide layer through the porous carbon nanotube yarn. Furthermore, the symmetric supercapacitor configuration demonstrated nearly $100 \%$ capacity retention at a bending angle of $150^{\circ}$.
\end{abstract}

\section{Introduction}

Among the many types of energy storage devices, electrochemical supercapacitors (SCs) are characterized by fast charge-discharge processes, a high power density, and a long cycle life [1,2]. With the broadening of their application range, such as in microgrid systems or wave-energy converter systems, the energy storage capability, mechanical flexibility, and robustness of electrochemical SCs need to be improved $[3,4]$. The elementary structure forming macroscopic SC devices can be quasi-one-dimensional (1D) such as a fiber, two-dimensional (2D) such as a sheet or paper, or three-dimensional (3D) with a porous network. In particular, in wearable and flexible electronic devices, 1D SCs have unique structural advantages such as those reported for a carbon nanotube (CNT) yarn [5-9]. The inherent mechanical flexibility, high toughness, and good thermal and electrical properties of a CNT yarn have been considered essential factors for high-performance energy storage devices [10-12].
However, owing to its non-Faradaic operational principle using an electrical double layer (EDL), the energy storage capability of a supercapacitor CNT yarn is inevitably limited. Therefore, the incorporation of Faradaic electrode materials (or pseudo-capacitive materials with electrochemical processes related to the reversible redox reaction of ions) has been investigated. For example, the addition of $\mathrm{MnO}_{2}$ or $\mathrm{TiO}_{2}$ particles to a supercapacitor CNT yarn showed the possibility of overcoming the limitations of EDL-based operation [13-16]. Particularly, pseudo-capacitive materials with a core/shell hybrid nanostructure have been explored, including $\mathrm{Co}_{3} \mathrm{O}_{4} / \mathrm{MnO}_{2}, \mathrm{Co}_{3} \mathrm{O}_{4} / \mathrm{NiO}$, and $\mathrm{ZnO} / \mathrm{NiO}$. These materials utilize the synergistic effects between the constituent materials to enhance the energy storage performance $[14,17]$. The recently reported combination of nickel-cobalt layered double hydroxide ( $\mathrm{NiCo}-\mathrm{OH})$ and nanostructured $\mathrm{ZnO}$ is another example of pseudo-capacitive core/shell hybrid nanostructures [18,19].

In this study, we propose a method for systematically combining pseudo-capacitive materials and CNT yarn to realize a high-capacitance

\footnotetext{
* Corresponding author. Department of Energy Science, Sungkyunkwan University, Suwon, 16419, Republic of Korea.

** Corresponding author.

E-mail addresses: baejh2k@gachon.ac.kr (J. Bae), energy.suh@skku.edu (D. Suh).
} 
flexible energy storage yarn. In the first stage, the surface area of a dryspun pristine CNT yarn used as a template is greatly increased by completely covering with a $\mathrm{ZnO}$ nanorod (ZnO-NR) forest. Then, the surface of the ZnO-NRs on the CNT yarn is covered with NiCo-OH flakes as an active material layer. The two-step process can be understood by the quantitative increase in surface area in the first step, which resulted from the physical surface area enhancement using $\mathrm{ZnO}-\mathrm{NR}$, and the qualitative increase in capacitance in the second step caused by the addition of a Faradaic charge-storage material on the expanded surface. The good electrochemical performance and superior mechanical flexibility upon bending as well as the reliability upon cycling confirms the feasibility of using the flexible fiber-shaped composite energy storage yarn in wearable electronic devices.

\section{Experimental method}

\subsection{Material synthesis}

\subsubsection{Preparation of CNT yarn}

A single-layer CNT sheet was pulled mechanically from a vertically grown spinnable CNT forest (purchased from A-Tech System Ltd., Incheon, Korea), and approximately 10-15 layers of CNT sheet were stacked using a U-shaped rotating jig, followed by the twisting of the CNT sheet layers to form a yarn with 100-200 turns per $6 \mathrm{~cm}$. The details of the formation of twisted CNT yarn and its properties were reported previously [20]. The yarn was used as a template for the growth of ZnO-NRs.

\subsubsection{Preparation of $\mathrm{ZnO}-\mathrm{NRs}$}

A ZnO-NR forest was grown from $\mathrm{ZnO}$ seed layers by a hydrothermal method involving the following two steps. 1) Fabrication of $\mathrm{ZnO}$ seed layer: a solution of $15 \mathrm{mM}$ zinc acetate dihydrate $\left(\mathrm{Zn}\left(\mathrm{CH}_{3} \mathrm{COO}\right)_{2} \cdot 2 \mathrm{H}_{2} \mathrm{O}\right.$; $\geq 98 \%)$ was prepared in anhydrous ethanol $\left(\mathrm{C}_{2} \mathrm{H}_{5} \mathrm{OH} ; \geq 99.5 \%\right)$. The mixture was dropped on a CNT yarn, dried in air, and placed onto a hot plate at $150{ }^{\circ} \mathrm{C}$ for $10 \mathrm{~min}$. The step was repeated two times to homogeneously diffuse the solution throughout the yarn. Then, the yarn was annealed at $350{ }^{\circ} \mathrm{C}$ for $4 \mathrm{~h}$ in an argon environment to form $\mathrm{ZnO}$ nuclei. In the case of a Pt wire (Sigma-Aldrich, 99.99\% trace metals basis, $\mathrm{d}=75 \mu \mathrm{m}$ ), pretreatment was first conducted by following a previously reported procedure [21]. The Pt wire was cleaned with ethanol and deionized (DI) water sequentially, followed by polishing with $0.05 \mathrm{mM}$ aluminum slurry. Finally, the substrate (CNT yarn or Pt wire) was dipped into a piranha solution for $10 \mathrm{~min}$. The $\mathrm{ZnO}$ seed layer was fabricated by spin coating the $15 \mathrm{mM} \mathrm{Zn}\left(\mathrm{CH}_{3} \mathrm{COO}\right)_{2}$ solution in ethanol using a centrifuge at $6000 \mathrm{rpm}$ for several hours, and annealing the substrate at $350{ }^{\circ} \mathrm{C}$. 2) Growth of $\mathrm{ZnO}$ nanorods: A mixture of $25 \mathrm{mM}$ zinc nitrate hexahydrate $\left(\mathrm{Zn}\left(\mathrm{NO}_{3}\right)_{2} \cdot 6 \mathrm{H}_{2} \mathrm{O} ; 98 \%\right)$ and $25 \mathrm{mM}$ hexamethylenetetramine $\left(\left(\mathrm{CH}_{2}\right)_{6} \mathrm{~N}_{4} ; 99 \%\right)$ in $1: 1$ vol ratio was used as a precursor to grow the nanorods. The CNT yarn (or Pt wire) coated by the $\mathrm{ZnO}$ seed layer was dipped into this mixture and heated in an oven for 4,8 , and $12 \mathrm{~h}$ at $90^{\circ} \mathrm{C}$. Then, the sample was cleaned by rinsing with DI water several times and annealed in an oven for $2 \mathrm{~h}$ at $150^{\circ} \mathrm{C}$.

\subsubsection{Fabrication of $\mathrm{NiCo}-\mathrm{OH}$}

A previously reported hydrothermal method for the synthesis of NiCo-OH [18] was modified and optimized in this work to successfully fabricate NiCo-OH on the ZnO-NR/CNT-yarn and ZnO-NR/Pt-wire. A NiCo precursor was prepared by mixing cobalt nitrate hexahydrate (Co $\left.\left(\mathrm{NO}_{3}\right)_{2} \cdot 6 \mathrm{H}_{2} \mathrm{O}\right)$ and nickel nitrate hexahydrate $\left(\mathrm{Ni}\left(\mathrm{NO}_{3}\right)_{2} \cdot 6 \mathrm{H}_{2} \mathrm{O}\right)$ in $15 \mathrm{~mL}$ DI water in a mole ratio of $2: 1$. Then, $0.15 \mathrm{~mL}$ of $3 \% \mathrm{NH}_{3}$ solution was dropped very slowly under stirring. The solution color changed from pink to purple. The solution was stabilized for several hours, transferred to a $20 \mathrm{~mL}$ Teflon-lined autoclave, and the ZnO-NR-coated CNT yarn (or Pt wire) was dipped into it and heated in an oven at $90^{\circ} \mathrm{C}$ for $4 \mathrm{~h}$. Next, the yarn was taken out of the solution, rinsed carefully with DI water, and dried overnight at $60^{\circ} \mathrm{C}$.

\subsubsection{Fabrication of gel electrolyte}

A LiOH-poly(vinyl alcohol) (PVA) gel was employed as an electrolyte, which also served as a separator in a full-cell symmetric SC configuration. First, $1.5 \mathrm{~g}$ of LiOH (98\%) was added to $30 \mathrm{~mL}$ of DI water and stirred for $30 \mathrm{~min}$. Then, $3 \mathrm{~g}$ of PVA ( $99 \%$, average molecular weight: 89000-98000) was added, and the solution was stirred at $95{ }^{\circ} \mathrm{C}$ until it turned into a clear gel.

\subsection{Diameter and weight measurement, electrode contact, and assembly of symmetric supercapacitor}

Diameter (d) measurement: The diameters were measured using an optical microscope at eight positions and the average value was obtained. They were confirmed by SEM as well.

Weight measurement: The weight was measured using a microbalance (BM-22 Ion Micro Balance $(5.1 \mathrm{~g} / 22 \mathrm{~g} \times 0.001 \mathrm{mg} / 0.01 \mathrm{mg})$, A\&D Weighing) in a dry room. Only the mass of NiCo-OH layer was used to calculate the active mass of the material for gravimetric capacitance.

Electrode contact: In a three-electrode system, to facilitate good contact between the fiber electrode and the electrochemical test station, a clean small Teflon plane $(0.5 \times 7 \mathrm{~cm})$ was prepared as a stable holder. Then, a copper tape was used as a current collector, which was connected to the yarn electrode and laid on the Teflon plane. Thereafter, silver paste was applied to the interface between the copper tape and fiber electrode. Finally, the electrode was placed in the RRDE-3A vial cell, and the surface area of electrode is estimated by the equation, $A=$ $\pi \times l \times d$, where $l, d$, and $A$ are the length, diameter, and area of the fiber dipped into electrolyte, respectively.

Assembly of symmetric supercapacitor in a two-electrode system: A clean poly(ethylene terephthalate) (PET, $2 \mathrm{~cm} \times 3 \mathrm{~cm}$ ) film was used as a flexible substrate. First, two electrodes were immersed in the gel for $15 \mathrm{~min}$. After they were taken out of the gel and dried in air, both the electrodes were assembled in parallel and attached onto the PET substrate with a thin gel layer. Copper tape was used to make electrical contacts with the sample. The distance between the two electrodes was optimized as $50-100 \mu \mathrm{m}$.

\subsection{Electrode morphology analysis}

The surface morphology of the electrode was examined by fieldemission scanning electron microscopy (SEM) (JSM-7000F, JEOL, Japan). The cross section of the sample was prepared using a focused ion beam (FIB, JIB-4601F, JEOL, Japan). The crystal and phase structures were characterized by X-ray diffraction (XRD) (D8 ADVANCE, Bruker, Germany) analysis using $\mathrm{Cu} \mathrm{K} \alpha$ radiation $(\lambda=1.5418 \AA)$. Highresolution transmission electron microscopy (TEM) images were obtained using a Cs-corrected transmission electron microscope (JEMARM200F, JEOL, Japan). For XRD and TEM analyses, ZnO-NRs grown for $8 \mathrm{~h}$ were used as a reference.

\subsection{Electrochemical characterization}

\subsubsection{Electrochemical measurement}

Cyclic voltammetry (CV), galvanostatic charge-discharge measurements, and frequency-dependent impedance measurements were performed at room temperature using a CV measurement system (Bio-Logic SA, VMP3, France). The three-electrode configuration was used with $1 \mathrm{M} \mathrm{LiOH}$ as an electrolyte, wherein the working electrode was the CNT yarn, ZnO-NR/CNT-yarn, or NiCo-OH/ZnO-NR/CNT-yarn. The reference electrode and counter electrode were a saturated calomel electrode (SCE) and a Pt coil, respectively. The electrochemical measurements of the symmetric SCs were performed with a two-electrode cell configuration of the $\mathrm{CV}$ system, where $\mathrm{NiCo}-\mathrm{OH} / \mathrm{ZnO}-\mathrm{NR} / \mathrm{CNT}$-yarn was employed as both the working and counter electrodes located on a PET flexible substrate. The PVA-LiOH gel served as both an electrolyte and a separator, simultaneously. 


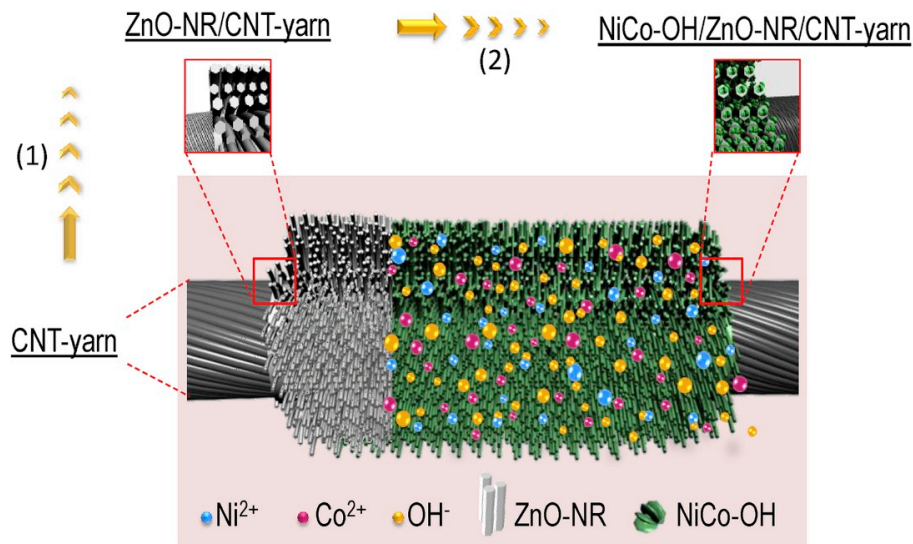

Fig. 1. High-performance quasi-1D supercapacitor fiber consisting of a NiCo-OH pseudo-capacitive layer, ZnO-NR forest, and dry-spun CNT yarn. (a) Schematic diagram of a NiCo-OH/ZnO-NR/CNTyarn fiber. (b) SEM image showing the surface and cross section of the synthesized $\mathrm{NiCo}-\mathrm{OH} / \mathrm{ZnO}$ NR/CNT-yarn fiber. The scale bar is $25 \mu \mathrm{m}$. (c) EDS elemental mapping images of $\mathrm{C}$ (green dots), Co (red dots), Ni (yellow dots), Zn (orange dots), and $\mathrm{O}$ (blue dots) in the NiCo-OH/ZnO-NR/CNT-yarn in the region enclosed by the white dash-dot rectangle in (b). (For interpretation of the references to color in this figure legend, the reader is referred to the Web version of this article.) b

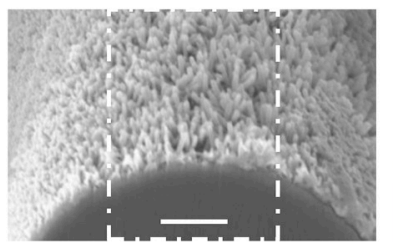

C

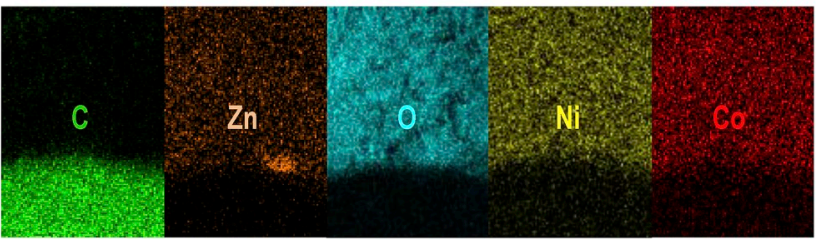

2.4.2. Calculations of capacitance, energy density, and power density

The specific capacitances estimated from the CV curves were calculated by the following equations:

$C_{M}=\frac{1}{M}\left[\frac{1}{v\left(V_{2}-V_{1}\right)} \int_{V_{1}}^{V_{2}} i(V) d V\right]$

$C_{A}=\frac{1}{A}\left[\frac{1}{v\left(V_{2}-V_{1}\right)} \int_{V_{1}}^{V_{2}} i(V) d V\right]$

Here, $C_{M}$ is the gravimetric (mass-specific) capacitance; $C_{A}$ is the areal (area-specific) capacitance; $M$ is the total mass of the active electrode materials; $v$. is the voltage scan rate; $i$ is the current (mA); $V_{1}$ and $V_{2}$ are the initial and final potentials, respectively; and $A$ is the area of the electrode dipped into an aqueous or a gel electrolyte.

The galvanostatic curves at varying current densities were used to calculate the areal energy density $(E)$ and areal power density $(P)$ of the SCs as follows:

$E=\frac{1}{A} \int i V(t) d t$

$P=\frac{E}{t}$

Here, $A$ is the total area of both the electrodes in a symmetric SC, and $t$ is the discharge time.

\subsection{Bending test}

The symmetric supercapacitor device was placed on a self-made bending tool, which was connected to a stepper motor. The tool can control the distance between the two ends of the device so that the device can be bent at different angles. The bending test is shown in video V1 provided in the Supplementary Information. To test at the angle of $150^{\circ}$, the device was kept at $150^{\circ}$ and then connected to the electrochemical station for measurement. In the bending $\left(150^{\circ}\right)$-unbending test, the device could be stably bent and unbent many times with the help of an automated (home-made) bending tool. After 1, 10, and 100 bending-unbending cycles, the device was connected to the VMP3 electrochemical station to obtain the CV curves.
Supplementary video related to this article can be found at https ://doi.org/10.1016/j.nanoen.2019.104198.

\section{Results and discussion}

Fig. 1a shows the schematic illustration for the fabrication of $\mathrm{ZnO}$ $\mathrm{NR} / \mathrm{NiCo}-\mathrm{OH}$ on the CNT yarn using the two-step process without any pretreatment of the CNT yarn. First, ZnO-NRs were grown on the surface of the CNT yarn after coating with a ZnO seed layer. Then, $\mathrm{NiCo-OH}$ was decorated on top of the ZnO-NR/CNT-yarn by the hydrothermal method. To clarify the structure of the as-prepared samples, the fiber electrode materials were analyzed by XRD. In Fig. S1a in the Supplementary Information (SI), the peak at $25.8^{\circ}$ in curve (a) is typically attributed to $\mathrm{CNT}$, while the other diffraction peaks can be indexed to the hexagonal ZnO crystal system (PDF Card No. 01-075-0576). Since all the peaks of ZnO-NR in the ZnO-NR/CNT-yarn fiber are sharp and narrow, the ZnONRs were considered to have a high purity and crystallinity. After coating NiCo-OH layers on the ZnO-NR/CNT-yarn, typical diffraction peaks were observed at $2 \theta$ values of $11.21^{\circ}, 22.79^{\circ}, 34.62^{\circ}$, and $60.65^{\circ}$ (curve (b)), which correspond to the $003,006,012$, and 110 planes, respectively. This proves the formation of a hydrotalcite-like $\mathrm{Ni}-\mathrm{Co}-\mathrm{OH}$ phase [22]. The result indicates that a NiCo-OH/ZnO-NR/CNT-yarn fiber electrode material can be successfully synthesized by the two-step hydrothermal process.

To study the structure in detail, TEM analysis was performed. Figs. S1b and S1c show the TEM images of ZnO-NR. The lattice spacing of $0.26 \mathrm{~nm}$ corresponds to the (002) plane of hexagonal $\mathrm{ZnO}$ (Fig. S1c). After the coating of NiCo-OH on the ZnO-NR, the surface became rough. The prepared NiCo-OH flake has a lattice spacing of $0.266 \mathrm{~nm}$, corresponding to the (012) plane (Fig. S1e).

Fig. $1 \mathrm{~b}$ and $\mathrm{c}$ shows the SEM image and the energy dispersive X-ray spectroscopy (EDS) mapping images of the NiCo-OH/ZnO-NR/CNTyarn, respectively. The sample cross section was prepared by Ga focused ion beam milling. The SEM image of the cross section and surface of the NiCo-OH/ZnO-NR/CNT-yarn (Fig. 1b) reveals that a ZnO-NR forest was successfully formed on the yarn's surface. The EDS elemental mapping images of $\mathrm{C}, \mathrm{Co}, \mathrm{Ni}, \mathrm{Zn}$, and $\mathrm{O}$ (Fig. 1c) confirm that the NiCo-OH layer is uniformly coated on the NiCo-OH/ZnO-NR/CNT-yarn surface, with no noticeable penetration of the constituent elements into the yarn except for carbon. 


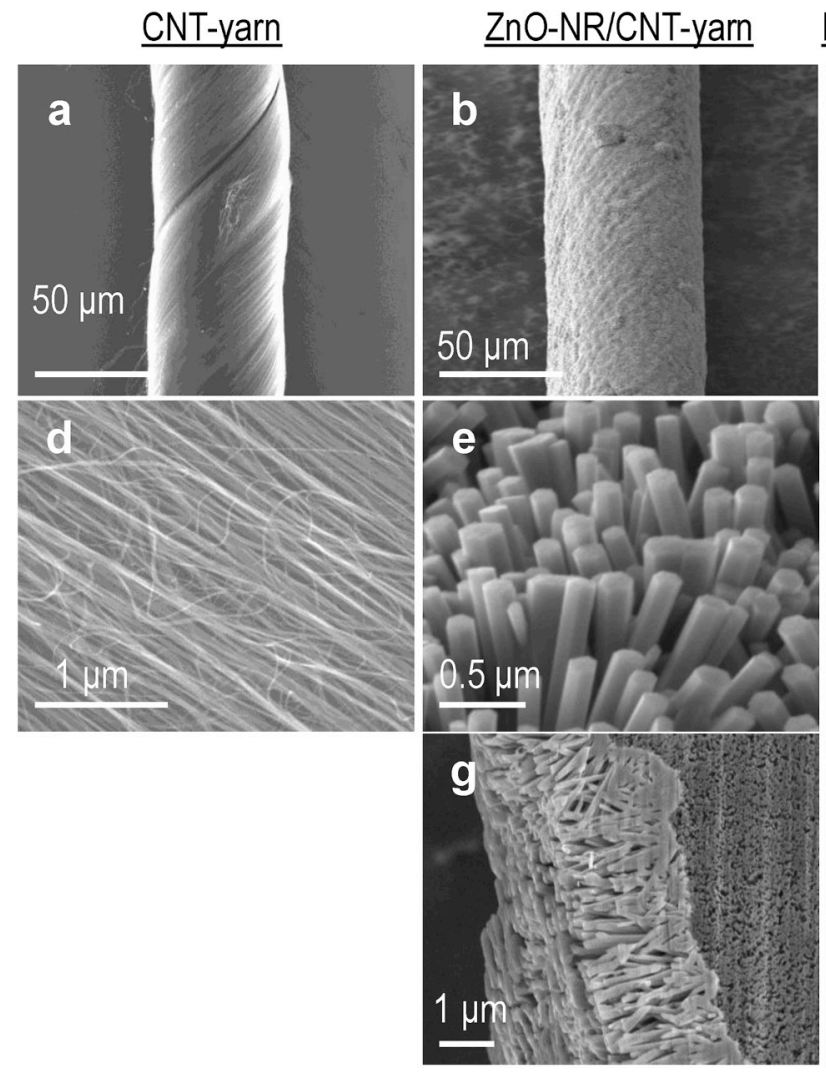

NiCo-OH/ZnO-NR/CNT-yarn

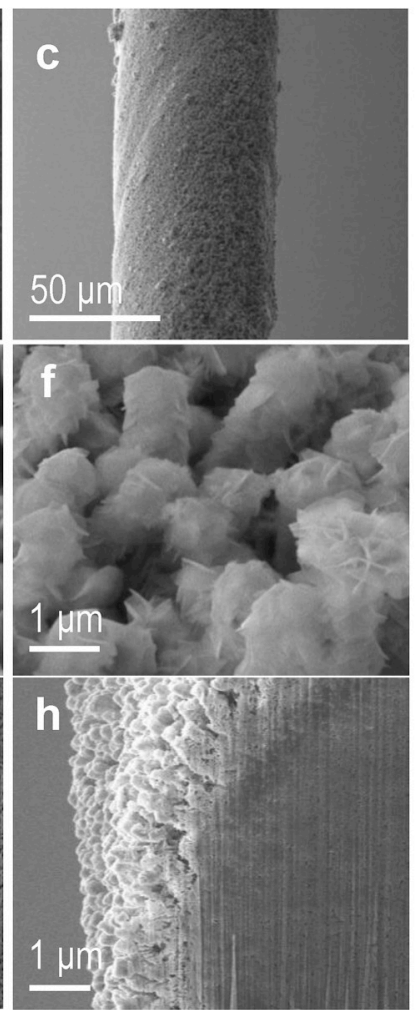

Fig. 2. SEM images of (a, d) pristine twisted CNT yarn, (b, e) ZnO-NRs on CNT yarn; and (c, f) hierarchical core-shell ZnO-NR/NiCo-OH on CNT yarn at low and high magnifications. $(\mathrm{g}, \mathrm{h})$ Internal core-shell structures before and after coating NiCo-OH on ZnO-NR/CNT-yarn.

a

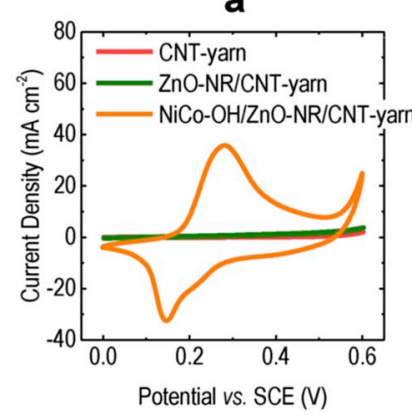

d

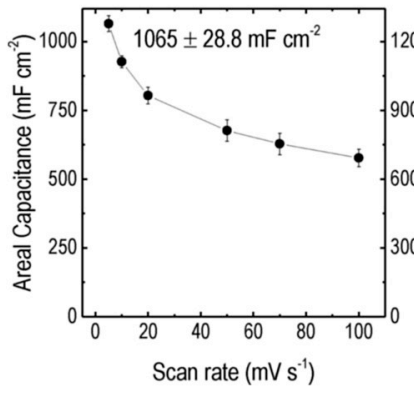

The SEM images in Fig. 2 show the morphologies of the hybrid flexible supercapacitor yarns and a bare CNT yarn. In the lowmagnification images (Fig. 2a-c), the overall shapes do not appear to be different for all the three samples. However, clear differences can be observed from the high-magnification images (Fig. 2d-f). Particularly, b

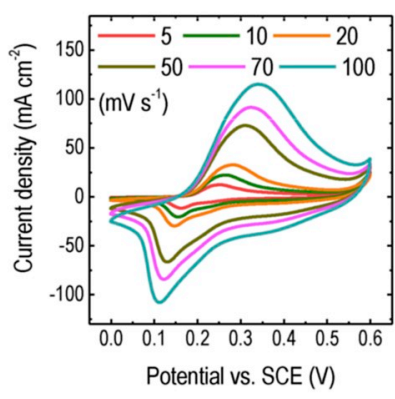

e

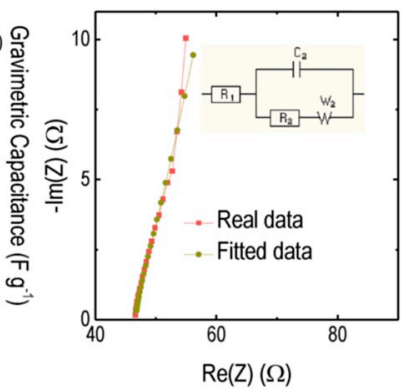

C

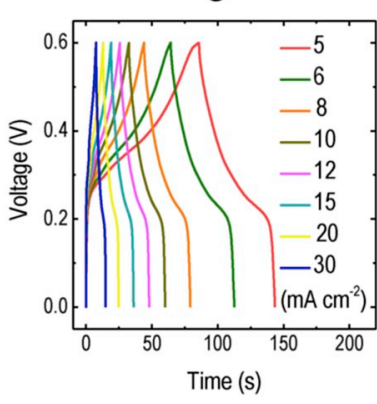

f

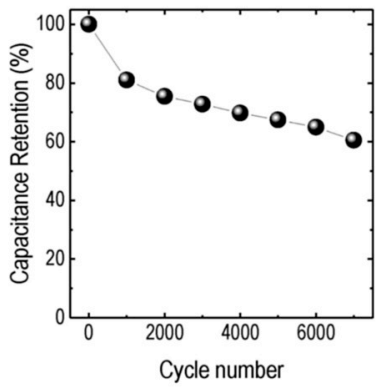

Fig. 3. Electrochemical performance of single electrode in $\mathrm{LiOH}(1 \mathrm{M})$ in a three-electrode system. (a) Cyclic voltammetry (CV) curves of CNT yarn, ZnO-NR/CNT-yarn, and NiCo-OH/ZnO-NR/ CNT-yarn at a scan rate of $20 \mathrm{mV} \mathrm{s}^{-1}$. (b) CV curves of NiCo-OH/ZnO-NR/CNT-yarn at different scan rates. (c) Galvanostatic charge-discharge curves of NiCo-OH/ZnO-NR/CNT-yarn at various current densities. (d) Capacitance of NiCo-OH/ZnO-NR/ CNT-yarn at various scan rates. (e) Nyquist plot and the equivalent circuit (inset) for $\mathrm{NiCo}-\mathrm{OH} /$ ZnO-NR/CNT-yarn. (f) Cycle stability at a current density of $30 \mathrm{~mA} \mathrm{~cm}^{-2}$ and capacitance retention after 7000 charge-discharge cycles. only aligned CNT nanofibers are observed in Fig. 2d, while a high density of vertically aligned ZnO-NRs homogeneously distributed on the CNT surface can be seen in Fig. 2e. The ZnO-NR with a hexagonal shape has an average diameter of around $200-300 \mathrm{~nm}$ and an average length of more than $1 \mu \mathrm{m}$ (Fig. 2e). Fig. $2 \mathrm{f}$ shows the NiCo-OH layers covering the 

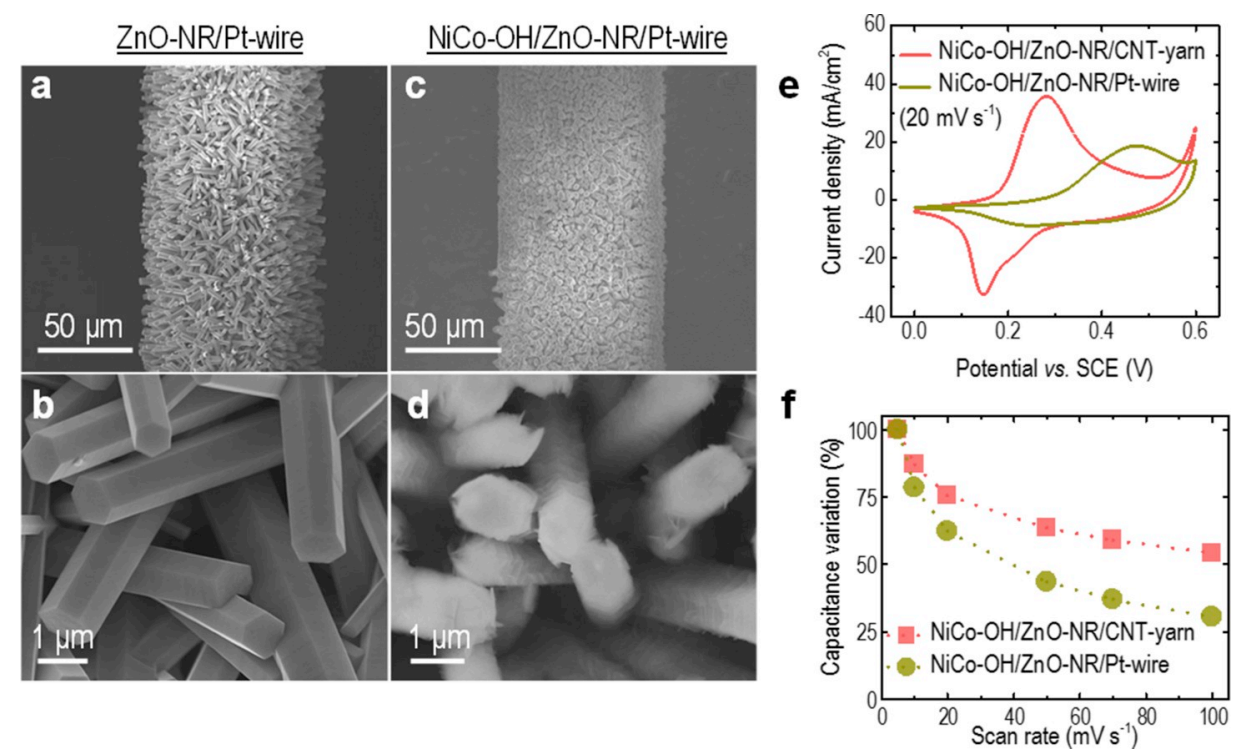

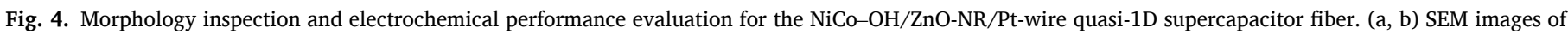

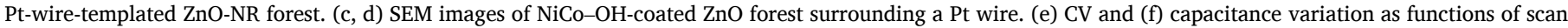
rate for the NiCo-OH/ZnO-NR/CNT-yarn electrode and $\mathrm{NiCo-OH/ZnO-NR/Pt-wire} \mathrm{electrode.}$

individual ZnO-NRs. Finally, the core-shell structure was confirmed from the high-magnification cross-sectional SEM images in Fig. $2 \mathrm{~g}$ and h.

The electrochemical performance of the hybrid SC yarn electrode is presented in Fig. 3. The CV curves of the bare CNT yarn, ZnO-NR/CNTyarn, and NiCo-OH/ZnO-NR/CNT-yarn obtained using a threeelectrode configuration with $\mathrm{LiOH}(1 \mathrm{M})$ electrolyte are shown in Fig. 3a. The maximum current density of NiCo-OH/ZnO-NR/CNT-yarn is approximately $35.7 \mathrm{~mA} \mathrm{~cm}^{-2}$, while those of the bare CNT yarn and ZnO-NR/CNT-yarn are $2.0 \mathrm{~mA} \mathrm{~cm}^{-2}$ and $3.7 \mathrm{~mA} \mathrm{~cm}^{-2}$, respectively. This indicates that the current density of $\mathrm{NiCo-OH/ZnO-NR/CNT-yarn}$ is approximately 10 times higher than those of the other electrodes. Moreover, redox reaction peaks can be clearly observed for the NiCo-OH/CNO-NR/CNT-yarn electrode. In other words, the decoration of $\mathrm{NiCo}-\mathrm{OH}$ significantly enhanced the electrochemical energy storage performance.

Fig. 3b demonstrates the CV curves of NiCo-OH/ZnO-NR/CNT-yarn at various scan rates $\left(5-100 \mathrm{mV} \mathrm{s}^{-1}\right)$. The curves show a typical SC behavior of increased current density proportional to the voltage scan rate. The prominent redox peaks, particularly at lower scan rates, indicate the pseudo-capacitive properties of NiCo-OH/ZnO-NR/CNT-yarn in the LiOH (1 M) electrolyte. The peaks are attributed to a combination of reversible Faradaic reactions of nickel hydroxide $\left(\mathrm{Ni}(\mathrm{OH})_{2}\right)$ and cobalt hydroxide $\left(\mathrm{Co}(\mathrm{OH})_{2}\right)$, followed by the following redox reactions [23]:

$\mathrm{Co}(\mathrm{OH})_{2}+\mathrm{OH}^{-} \rightleftharpoons \mathrm{CoOOH}+\mathrm{H}_{2} \mathrm{O}+\mathrm{e}^{-}$

$\mathrm{CoOOH}+\mathrm{OH}^{-} \rightleftharpoons \mathrm{CoO}_{2}+\mathrm{H}_{2} \mathrm{O}+\mathrm{e}^{-}$

$\mathrm{Ni}(\mathrm{OH})_{2}+\mathrm{OH}^{-} \rightleftharpoons \mathrm{NiOOH}+\mathrm{H}_{2} \mathrm{O}+\mathrm{e}^{-}$

Herein, only one pair of reduction and oxidation peaks was observed without additional redox peaks, unlike in a previous study [24]. These results indicate that $\mathrm{Ni}(\mathrm{OH})_{2}$ and $\mathrm{Co}(\mathrm{OH})_{2}$ are well mixed in the NiCo-OH [23].

Fig. 3c shows the galvanostatic charge-discharge curves of NiCo-OH/ZnO-NR/CNT-yarn, wherein the non-triangular shapes can be attributed to the effect of the pseudo-capacitive component of the NiC$\mathrm{o}-\mathrm{OH}$ layer. The areal and gravimetric capacitances of the $\mathrm{NiCo}-\mathrm{OH} /$ ZnO-NR/CNT-yarn are presented in Fig. 3d as a function of scan rate, which show that the inhomogeneous porosity distribution inside the electrode may result in a decrease in specific capacitance at high scan rates. The maximum capacitance of NiCo-OH/ZnO-NR/CNT-yarn $\left(1278 \mathrm{~F} \mathrm{~g}^{-1}\right)$ is much higher than those of the other $\mathrm{ZnO}$-incorporated systems, such as ZnO-CNT composites, ZnO-graphene composite, and ZnO-activated carbon composites [25-29].

Further, the frequency response of NiCo-OH/ZnO-NR/CNT-yarn was analyzed by electrochemical impedance spectroscopy (EIS) by applying a $10 \mathrm{mV}$ DC bias potential in the frequency range of $100 \mathrm{kHz}$ to $100 \mathrm{MHz}$; the results are shown in Fig. 3e. From the $\mathrm{x}$-axis intercept of the Nyquist plot obtained from the EIS measurements, the internal resistance of the hybrid SC is estimated to be approximately $46 \Omega$. The cycle stability test of NiCo-OH/ZnO-NR/CNT-yarn was performed at a current density of $30 \mathrm{~mA} \mathrm{~cm}^{-2}$, and the results are shown in Fig. 3f. The result indicates that the capacitance retention, defined as $\mathrm{C} / \mathrm{C}_{0}\left(\mathrm{C}_{0}\right.$ : initial capacitance), is $60.5 \%$ after 7000 charge-discharge cycles, and this is comparable to or better than those of NiCo-OH nanoflakes on a carbon fiber membrane, $\mathrm{NiCo}-\mathrm{OH}$-covered zinc tin oxide nanowires grown on stainless steel, and $\mathrm{Mn}_{3} \mathrm{O}_{4} / \mathrm{CNT}$ electrode [30-32].

To further understand the effect of $\mathrm{ZnO}$ nanostructure on the performance of the system, composites with different growth times of $\mathrm{ZnO}$ $(4,8$, and $12 \mathrm{~h})$ were prepared by the same protocol. The SEM images are shown in Figs. S3(b, e, h). As can be seen, at $4 \mathrm{~h}$, the sample has smaller and shorter rods than those in the $8 \mathrm{~h}$ sample. Although the rod size slightly changed at $12 \mathrm{~h}$ (Fig. S3h), the density of rods increased, which may reduce the space for the growth of $\mathrm{NiCo}-\mathrm{OH}$ and mass transport. Therefore, $8 \mathrm{~h}$ was considered the optimal growth condition. To evaluate the performance, we recorded the $\mathrm{CV}$ curves of each sample at various scan rates. As expected, the best performance was achieved at $8 \mathrm{~h}$ (Fig. S3j). The larger diameter and lesser density of rods at $8 \mathrm{~h}$ of growth introduce significant porosity in each rod, thereby providing sufficient diffusion paths for the ions in the electrolyte and facilitating reaction with the active materials of the NiCo-OH layer.

As observed in Fig. 3a, the high specific capacitance of NiCo-OH/ ZnO-NR/CNT-yarn primarily originates from the pseudo-capacitance behavior of the $\mathrm{NiCo}-\mathrm{OH}$ layer coated on the large surface of the $\mathrm{ZnO}$ NRs. Because the contribution of CNT yarn as a supercapacitive material appears negligible in this system, the advantage of the CNT yarn as a template for quasi-1D structures needs to be clarified. For this purpose, $\mathrm{NiCo}-\mathrm{OH} / \mathrm{ZnO}-\mathrm{NR} / \mathrm{Pt}$-wire, in which a Pt wire was used as a template instead of a CNT yarn for quasi-1D structures, was examined. Fig. 4 


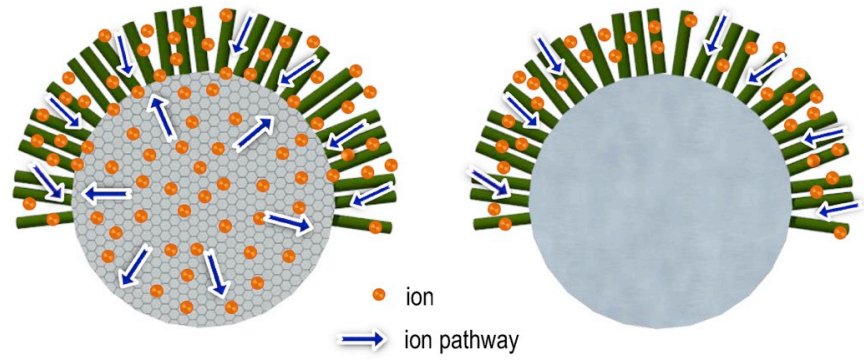

NiCo-OH/ZnO-NR/CNT-yarn

NiCo-OH/ZnO-NR/Pt-wire

Fig. 5. Illustrations representing the cross sections of NiCo-OH/ZnO-NR/CNTyarn electrode (left) and $\mathrm{NiCo-OH/ZnO-NR/Pt-wire} \mathrm{electrode} \mathrm{(right).} \mathrm{The}$ porous nature of the CNT yarn template facilitates ion movement through the quasi-1D template. The Pt wire does not have ion channels.

shows the morphology and device performance of the NiCo-OH/ZnONR/Pt-wire system. The shapes of ZnO-NRs on Pt wire (in Fig. 4a and b) and NiCo-OH-coated ZnO-NRs on Pt wire (in Fig. 4c and d) are similar to those of ZnO-NRs in the CNT yarn systems (Fig. 2). Moreover, the performance of the NiCo-OH/ZnO-NR/CNT-yarn system is better than that of the NiCo-OH/ZnO-NR/Pt-wire system having a similar size in terms of specific capacitance (Fig. 4e) and scan speed dependence (Fig. 4f).

The main difference between the Pt wire template and CNT yarn template can be considered in two aspects: the higher electrical conductivity of Pt wire, and the higher porosity of the CNT yarn. To explain the experimental data in Fig. $4 \mathrm{e}$ and $\mathrm{f}$, the effect of porosity of the CNT yarn template should be deeply considered. Because the NiCo-OHcoated $\mathrm{ZnO}-\mathrm{NR}$ forest layer provides an electrochemical surface area, the better performance of NiCo-OH/ZnO-NR/CNT-yarn than that of $\mathrm{NiCo}-\mathrm{OH} / \mathrm{ZnO}-\mathrm{NR} / \mathrm{Pt}$-wire can be ascribed to the enhanced ion accessibility in the case of CNT yarn template, which has a larger active surface area. Owing to the cylindrical shape of the $\mathrm{ZnO}-\mathrm{NR} / \mathrm{NiCo}-\mathrm{OH}$ forest surrounding the quasi-1D template, as shown in Fig. 1, the region near the roots of the ZnO-NRs may not be easily accessible to the ions in the electrolyte approaching from the outside, especially in the case of $\mathrm{Pt}$ wire template. However, when the CNT yarn template was employed, the inherently formed ion channels through the connected pores inside the CNT yarn facilitated the accessibility of ions to the $\mathrm{NiCo-OH} / \mathrm{ZnO}$ NR layer, which resulted in a larger specific capacitance. This process is schematically illustrated in Fig. 5.

For the flexibility and reliability test, a symmetric two-electrode cell consisting of a pair of parallelly arranged $\mathrm{NiCo-OH/ZnO-NR/CNT-yarn}$ electrodes and an ion-gel electrolyte was fabricated, and is shown in Fig. S6 in the SI. The CV curves in Fig. S7 in the SI confirm the operation of a SC energy storage device. As can be seen in Fig. 6, no variation is observed in the CV curves obtained at various bending angles, including at $150^{\circ}$, which shows the excellent mechanical flexibility of the device. The CV curves of the device bent at $150^{\circ}$ at $1^{\text {st }}$ and $1000^{\text {th }}$ cycles obtained at a scan rate of $100 \mathrm{mV} \mathrm{s}^{-1}$ are shown in Fig. S9b. The capacitance difference was calculated to be approximately $75 \%$ after 1000 cycles, as shown in Fig. 6c.

Using equations ( 3 ) and (4), we calculated the areal energy density and areal power density, and the Ragone plot of our device is displayed in Fig. 6d. The areal energy density and areal power density are $0.897-1.38 \mu \mathrm{Wh} \mathrm{cm}^{-2}$ and $117-647 \mu \mathrm{W} \mathrm{cm}^{-2}$, respectively. The maximum areal power density is higher than that of a polyester@MXene nanofiber-coated yarn $\left(390 \mu \mathrm{W} \mathrm{cm}{ }^{-2}\right)$ [33] and graphene fiber-based SCs prepared using plasma treatment $\left(20 \mu \mathrm{W} \mathrm{cm}^{-2}\right)$ [34]. The areal energy density is comparable to that of a $\mathrm{Co}_{3} \mathrm{O}_{4} @ \mathrm{CNT}$ composite yarn $\left(1.1 \mu \mathrm{Wh} \mathrm{cm}{ }^{-2}\right)$ [35], and five-fold greater than that of a CNT fiber stretchable in PVA- $\mathrm{H}_{2} \mathrm{SO}_{4}\left(0.226 \mu \mathrm{Wh} \mathrm{cm}{ }^{-2}\right)$ [11].

For a more detailed comparison of our results with those of other fiber-shaped SCs, we summarize some representative results of SC a

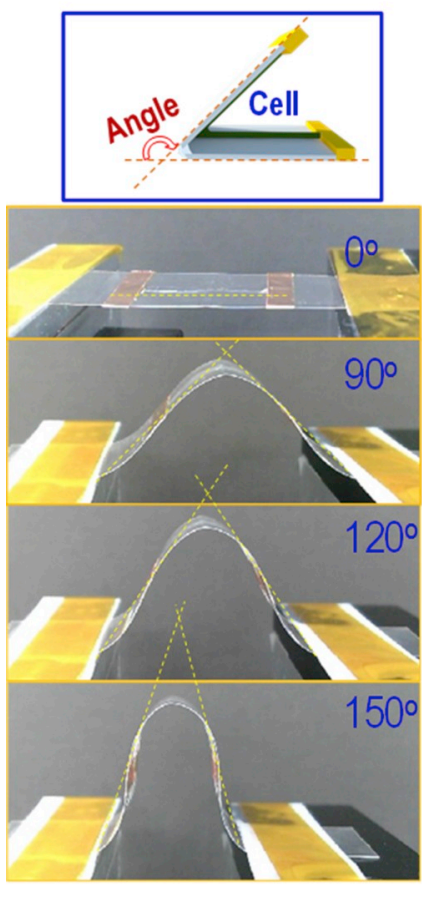

b

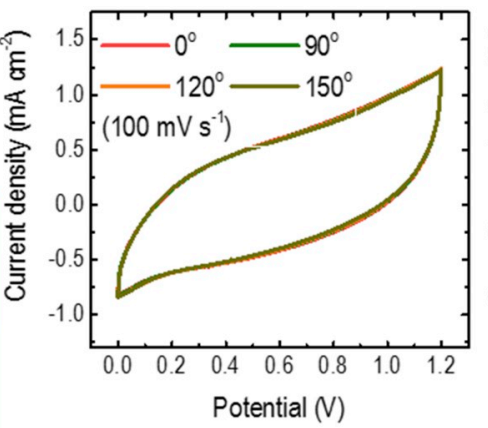

d

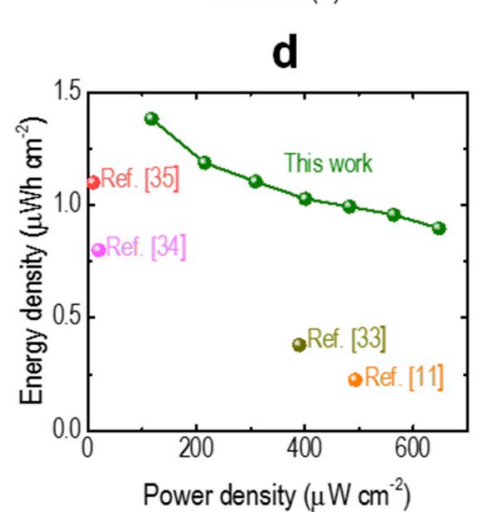

C

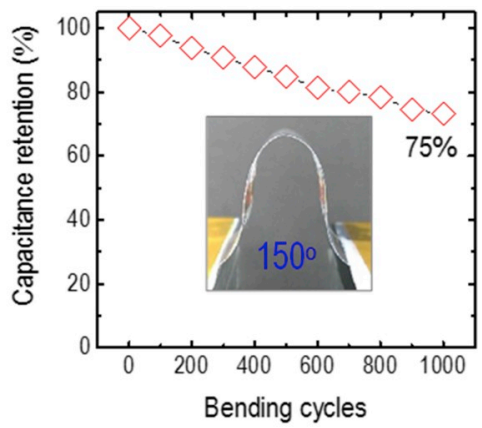

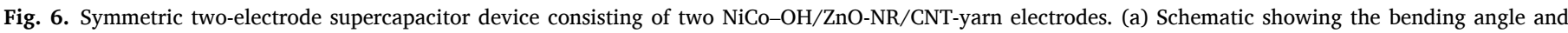

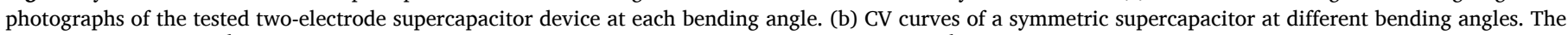

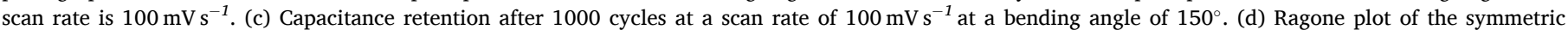
supercapacitor showing the areal energy density and areal power density with maximum values of $1.38 \mu \mathrm{Wh} \mathrm{cm}^{-2}$ and $647 \mu \mathrm{W} \mathrm{cm}^{-2}$, respectively. 
electrode materials based on CNT or NiCo-OH in Table S1 in the SI [11, 18,30,31,35-45]. The results indicate that our approach to the systematic enhancement of capacitance in a fiber-shaped NiCo-OH/ZnO-NR/CNT-yarn SC electrode is highly promising in terms of not only specific capacitance, but also durability and mechanical flexibility.

\section{Conclusions}

As a candidate for quasi-1D fiber-shaped energy storage devices, a flexible electrochemical SC yarn consisting of NiCo-OH, ZnO-NR, and dry-spun CNT yarn was fabricated by a two-step hydrothermal process without any treatment of the CNT yarn. The NiCo-OH/ZnO-NR/CNTyarn fiber in a three-electrode system yielded a high specific capacitance of $1065 \mathrm{mF} \mathrm{cm}^{-2}\left(1278 \mathrm{~F} \mathrm{~g}^{-1}\right)$ and exhibited good charge-discharge cycling stability (60.5\% capacitance retention after 7000 cycles). In addition, the symmetric two-electrode system showed excellent flexibility without any significant change in capacitance when bent at $150^{\circ}$. Furthermore, the role of ion movement channels through the CNT yarn was clearly analyzed. The results indicate that our approach of systematic increase in capacitance, which was accomplished by surface area increment through $\mathrm{ZnO}-\mathrm{NR}$ growth on a quasi-1D template followed by the coating of a pseudo-capacitive NiCo-OH layer, can be promising for the development of high-performance fiber-shaped energy storage devices.

\section{Notes}

The authors declare no competing financial interest.

\section{Acknowledgments}

This work was supported by the Korea Electric Power Corporation (No. R18XA06-54), and by the National Research Foundation of Korea funded by the Ministry of Science and ICT (No. NRF2018K1A4A3A01064272 and NRF-2017R1D1A1B03032466), Republic of Korea.

\section{Appendix A. Supplementary data}

Supplementary data to this article can be found online at https://doi. org/10.1016/j.nanoen.2019.104198.

\section{References}

[1] P. Simon, Y. Gogotsi, Materials for electrochemical capacitors, Nat. Mater. 7 (2008) 845-854. https://doi:10.1038/nmat2297.

[2] A. Burke, Ultracapacitors: why, how, and where is the technology, J. Power Sources 91 (2000) 37-50, https://doi.org/10.1016/S0378-7753(00)00485-7.

[3] F.A. Inthamoussou, J. Pegueroles-Queralt, F.D. Bianchi, Control of a supercapacitor energy storage system for microgrid applications, IEEE Trans. Energy Convers. 28 (2013) 690-697, https://doi.org/10.1109/TEC.2013.2260752.

[4] D.B. Murray, J.G. Hayes, D.L. O'Sullivan, M.G. Egan, Supercapacitor testing for power smoothing in a variable speed offshore wave energy converter, IEEE J. Ocean. Eng. 37 (2012) 301-308, https://doi.org/10.1109/JOE.2012.2188157.

[5] L. Dong, C. Xu, Y. Li, Z.-H. Huang, F. Kang, Q.-H. Yang, X. Zhao, Flexible electrodes and supercapacitors for wearable energy storage: a review by category, J. Mater. Chem. 4 (2016) 4659-4685, https://doi.org/10.1039/C5TA10582J.

[6] L. Kou, T. Huang, B. Zheng, Y. Han, X. Zhao, K. Gopalsamy, H. Sun, C. Gao, Coaxial wet-spun yarn supercapacitors for high-energy density and safe wearable electronics, Nat. Commun. 5 (2014) 3754, https://doi.org/10.1038/ncomms4754.

[7] X. Chen, L. Qiu, J. Ren, G. Guan, H. Lin, Z. Zhang, P. Chen, Y. Wang, H. Peng, Nove electric double-layer capacitor with a coaxial fiber structure, Adv. Mater. 25 (2013) 6436-6441, https://doi.org/10.1002/adma.201301519.

[8] D. Zhang, Y. Wu, T. Li, Y. Huang, A. Zhang, M. Miao, High performance carbon nanotube yarn supercapacitors with a surface-oxidized copper current collector, ACS Appl. Mater. Interfaces 7 (2015) 25835-25842, https://doi.org/10.1021/ acsami.5b08110.

[9] J.A. Lee, M.K. Shin, S.H. Kim, H.U. Cho, G.M. Spinks, G.G. Wallace, M.D. Lima, X. Lepro, M.E. Kozlov, R.H. Baughman, S.J. Kim, Ultrafast charge and discharge biscrolled yarn supercapacitors for textiles and microdevices, Nat. Commun. 4 (2013) 1970, https://doi.org/10.1038/ncomms2970.
[10] T. Chen, L. Dai, Flexible supercapacitors sased on carbon nanomaterials, J. Mater. Chem. 2 (2014) 10756-10775, https://doi.org/10.1039/C4TA00567H.

[11] P. Xu, T. Gu, Z. Cao, B. Wei, J. Yu, F. Li, J.-H. Byun, W. Lu, Q. Li, T.-W. Chou, Carbon nanotube fiber based stretchable wire-shaped supercapacitors, Adv. Energy Mater. 4 (2014) 1300759, https://doi.org/10.1002/aenm.201300759.

[12] S.T. Senthilkumar, Y. Wang, H. Huang, Advances and prospects of fiber supercapacitors, J. Mater. Chem. 3 (2015) 20863-20879, https://doi.org/ 10.1039/C5TA04731E.

[13] W. Zeng, L. Wang, H. Shi, G. Zhang, K. Zhang, H. Zhang, F. Gong, T. Wang, $\mathrm{H}$. Duan, Metal-organic-framework-derived ZnO@C@ $\mathrm{NiCo}_{2} \mathrm{O}_{4}$ core-shell structures as an advanced electrode for high-performance supercapacitors, J. Mater. Chem. 4 (2016) 8233-8241, https://doi.org/10.1039/C6TA01510G.

[14] J. Liu, J. Jiang, C. Cheng, H. Li, J. Zhang, H. Gong, H.J. Fan, $\mathrm{Co}_{3} \mathrm{O}_{4}$ nanowire@ $\mathrm{MnO}_{2}$ ultrathin nanosheet core/shell arrays: a new class of high-performance pseudocapacitive materials, Adv. Mater. 23 (2011) 2076-2081, https://doi.org/ 10.1002/adma.201100058.

[15] F. Su, M. Miao, Asymmetric carbon nanotube- $\mathrm{MnO}_{2}$ two-ply yarn supercapacitors for wearable electronics, Nanotechnology 25 (2014) 135401, https://doi.org/ 10.1088/0957-4484/25/13/135401.

[16] T. Li, Y. Wu, Q. Wang, D. Zhang, A. Zhang, M. Miao, $\mathrm{TiO}_{2}$ crystalline structure and electrochemical performance in two-ply yarn $\mathrm{CNT} / \mathrm{TiO}_{2}$ symmetric supercapacitors, J. Mater. Sci. 52 (2017) 7733-7743, https://doi.org/10.1007/ s10853-017-1033-6.

[17] X. Xia, J. Tu, Y. Zhang, X. Wang, C. Gu, X.-b. Zhao, H.J. Fan, High-quality metal oxide core/shell nanowire arrays on conductive substrates for electrochemical energy storage, ACS Nano 6 (2012) 5531-5538, https://doi.org/10.1021/ nn301454q.

[18] N.T. Trang, H.V. Ngoc, N. Lingappan, D.J. Kang, A comparative study of supercapacitive performances of nickel cobalt layered double hydroxides coated on $\mathrm{ZnO}$ nanostructured arrays on textile fibre as electrodes for wearable energy storage devices, Nanoscale 6 (2014) 2434-2439, https://doi.org/10.1039/ C3NR04764D.

[19] I. Shakir, M. Shahid, U.A. Rana, I.M.A. Nashef, R. Hussain, Nickel-cobalt layered double hydroxide anchored zinc oxide nanowires grown on carbon fiber cloth for high-performance flexible pseudocapacitive energy storage devices, Electrochim. Acta 129 (2014) 28-32, https://doi.org/10.1016/j.electacta.2014.02.082.

[20] T.K. Truong, Y. Lee, D. Suh, Multifunctional characterization of carbon nanotube sheets, yarns, and their composites, Curr. Appl. Phys. 16 (2016) 1250-1258, https://doi.org/10.1016/j.cap.2016.04.023.

[21] R. Dhull, K. Rathee, S. Singh, Synthesis of zinc oxide nanorods on platinum wire \& fabrication of triglyceride biosensor, J. Pharm. Sci. 8 (2016) 170-181. http://sch olarsresearchlibrary.com/archive.html.

[22] M. Jing, H. Hou, C.E. Banks, Y. Yang, Y. Zhang, X. Ji, Alternating voltage introduced nico double hydroxide layered nanoflakes for an asymmetric supercapacitor, ACS Appl. Mater. Interfaces 7 (2015) 22741-22744, https://doi. org/10.1021/acsami.5b05660.

[23] Y. Cheng, H. Zhang, C.V. Varanasi, J. Liu, Improving the performance of cobalt-nickel hydroxide-based self-supporting electrodes for supercapacitors using accumulative approaches, Energy Environ. Sci. 6 (2013) 3314, https://doi.org/ 10.1039/C3EE41143E.

[24] Y. Tao, L. Zaijun, L. Ruiyi, N. Qi, K. Hui, N. Yulian, L. Junkang, Nickel-cobalt double hydroxides microspheres with hollow interior and hedgehog-like exterior structures for supercapacitors, J. Mater. Chem. 22 (2012) 23587, https://doi.org/ 10.1039/C2JM35263J.

[25] Y. Zhang, X. Sun, L. Pan, H. Li, Z. Sun, C. Sun, B.K. Tay, Carbon nanotube-zinc oxide electrode and gel polymer electrolyte for electrochemical supercapacitors, J. Alloy. Comp. 480 (2009) L17-L19, https://doi.org/10.1016/j. jallcom.2009.01.114.

[26] Y. Zhang, X. Sun, L. Pan, H. Li, Z. Sun, C. Sun, B.K. Tay, Carbon nanotube-ZnO nanocomposite electrodes for supercapacitors, Solid State Ion. 180 (2009) 1525-1528, https://doi.org/10.1016/j.ssi.2009.10.001.

[27] T. Lu, Y. Zhang, H. Li, L. Pan, Y. Li, Z. Sun, Electrochemical behaviors of graphene- $\mathrm{ZnO}$ and graphene- $\mathrm{SnO}_{2}$ composite films for supercapacitors, Electrochim. Acta 55 (2010) 4170-4173, https://doi.org/10.1016/j. electacta.2010.02.095.

[28] T. Lu, L. Pan, H. Li, G. Zhu, T. Lv, X. Liu, Z. Sun, T. Chen, D.H.C. Chua, Microwaveassisted synthesis of graphene-ZnO nanocomposite for electrochemical supercapacitors, J. Alloy. Comp. 509 (2011) 5488-5492, https://doi.org/10.1016/ j.jallcom.2011.02.136.

[29] M. Selvakumar, D. Krishna Bhat, A. Manish Aggarwal, S. Prahladh Iyer, G. Sravani, Nano ZnO-activated carbon composite electrodes for supercapacitors, Phys. B (Amsterdam, Neth.) 405 (2010) 2286-2289, https://doi.org/10.1016/j. physb.2010.02.028.

[30] F. Lai, Y. Huang, Y.-E. Miao, T. Liu, Controllable preparation of multi-dimensional hybrid materials of nickel-cobalt layered double hydroxide nanorods/nanosheets on electrospun carbon nanofibers for high-performance supercapacitors, Electrochim. Acta 174 (2015) 456-463, https://doi.org/10.1016/j. electacta.2015.06.031.

[31] X. Wang, A. Sumboja, M. Lin, J. Yan, P.S. Lee, Enhancing electrochemical reaction sites in nickel-cobalt layered double hydroxides on zinc tin oxide nanowires: a hybrid material for an asymmetric supercapacitor device, Nanoscale 4 (2012) 7266-7272, https://doi.org/10.1039/C2NR31590D.

[32] G. An, P. Yu, M. Xiao, Z. Liu, Z. Miao, K. Ding, L. Mao, Low-temperature synthesis of $\mathrm{Mn}_{3} \mathrm{O}_{4}$ nanoparticles loaded on multi-walled carbon nanotubes and their application in electrochemical capacitors, Nanotechnology 19 (2008) 275709, https://doi.org/10.1088/0957-4484/19/27/275709. 
[33] W. Shaoa, M. Tebyetekerwaa, I. Marriama, W. Lib, Y. Wuc, e. Shengjie Pengd, S. Ramakrishnae, S. Yang, M. Zhu, Polyester@MXene nanofibers-based yarn electrodes, J. Power Sources 396 (2018) 683-690. https://doi.org/10.1016/j. jpowsour.2018.06.084.

[34] J. Meng, W. Nie, K. Zhang, F. Xu, X. Ding, S. Wang, Y. Qiu, Enhancing electrochemical performance of graphene fiber-based supercapacitors by plasma treatment, ACS Appl. Mater. Interfaces 10 (2018) 13652-13659, https://doi.org/ 10.1016/j.jpowsour.2018.06.084.

[35] F. Su, X. Lv, M. Miao, High-performance two-ply yarn supercapacitors based on carbon nanotube yarns dotted with $\mathrm{Co}_{3} \mathrm{O}_{4}$ and NiO nanoparticles, Small 11 (2015) 854-861, https://doi.org/10.1002/smll.201401862.

[36] F. Su, M. Miao, Flexible, high performance two-ply yarn supercapacitors based on irradiated carbon nanotube yarn and PEDOT/PSS, Electrochim. Acta 127 (2014) 433-438, https://doi.org/10.1016/j.electacta.2014.02.064.

[37] P. Shi, L. Li, L. Hua, Q. Qian, P. Wang, J. Zhou, G. Sun, W. Huang, Design of amorphous manganese oxide@multiwalled carbon nanotube fiber for robust solidstate supercapacitor, ACS Nano 11 (2017) 444-452, https://doi.org/10.1021/ acsnano.6b06357.

[38] K. Wang, Q. Meng, Y. Zhang, Z. Wei, M. Miao, High-performance two-ply yarn supercapacitors based on carbon nanotubes and polyaniline nanowire arrays, Adv. Mater. 25 (2013) 1494-1498, https://doi.org/10.1002/adma.201204598.

[39] J. Sun, Y. Huang, C. Fu, Z. Wang, Y. Huang, M. Zhu, C. Zhi, H. Hu, Highperformance stretchable yarn supercapacitor based on PPy@CNTs@Urethane elastic fiber core spun yarn, Nano Energy 27 (2016) 230-237, https://doi.org/ 10.1016/j.nanoen.2016.07.008.

[40] O. Yang, X.T. Zhang, M.Y. Zhang, Y. Gao, H. Gao, X.C. Liu, H. Liu, K.W. Wong, W. M. Lau, Rationally designed hierarchical $\mathrm{MnO}_{2}$-shell/ZnO-nanowire/carbon-fabric for high-performance supercapacitor electrodes, J. Power Sources 272 (2014) 654-660, https://doi.org/10.1016/j.jpowsour.2014.09.001.

[41] P. Yang, X. Xiao, Y. Li, Y. Ding, P. Qiang, X. Tan, W. Mai, Z. Lin, W. Wu, T. Li, H. Jin, P. Liu, J. Zhou, C.P. Wong, Z.L. Wang, Hydrogenated ZnO core shell nanocables for flexible supercapacitors and self-powered systems, ACS Nano 7 (2013), https://doi.org/10.1021/nn306044d, 2617-1626.

[42] R.R. Salunkhe, K. Jang, S.-w. Lee, S. Yu, H. Ahn, Binary metal hydroxide nanorods and multi-walled carbon nanotube composites for electrochemical energy storage applications, J. Mater. Chem. 22 (2012) 21630-21635, https://doi.org/10.1039/ C2JM32638H.

[43] X. Cai, X. Shen, L. Ma, Z. Ji, C. Xu, A. Yuan, Solvothermal synthesis of nico-layered double hydroxide nanosheets decorated on rGO sheets for high performance supercapacitor, Chem. Eng. J. 268 (2015) 251-259, https://doi.org/10.1016/j. cej.2015.01.072

[44] S.C. Sekhar, G. Nagaraju, J.S. Yu, Conductive silver nanowires-fenced carbon cloth fibers-supported layered double hydroxide nanosheets as a flexible and binder-free electrode for high-performance asymmetric supercapacitors, Nano Energy 36 (2017) 58-67, https://doi.org/10.1016/j.nanoen.2017.04.019.

[45] H. Wu, Z. Lou, H. Yang, G. Shen, A flexible spiral-type supercapacitor based on $\mathrm{ZnCO}_{2} \mathrm{O}_{4}$ nanorod electrodes, Nanoscale 7 (2015) 1921-1926, https://doi.org/ 10.1039/C4NR06336H.

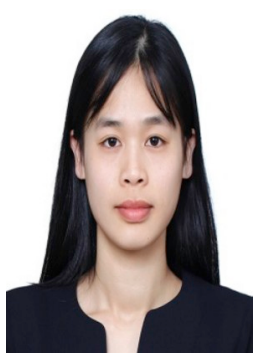

Thi Suong Le is currently a graduate student at Sungkyunkwan University, Korea. Her research interest is multi-walled carbon nanotube sheets and yarns for electrochemical applications.

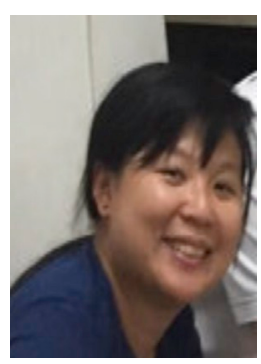

Thuy Kieu Truong received her Ph.D. degree from Sung kyunkwan University in 2019. Presently, she is a researcher in the Institute of Physics, Vietnam Academy of Science and Technology. Her research interests are multi-walled carbon nanotubes for liquid crystals and actuators.

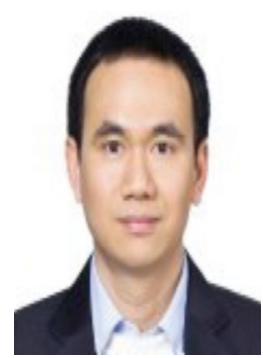

Van Ngoc Huynh received his Ph.D. degree from Sungkyunkwan University in 2015. Presently, he is a postdoctoral fellow in the Technical University of Denmark, Denmark. His research interests are two-dimensional material devices.

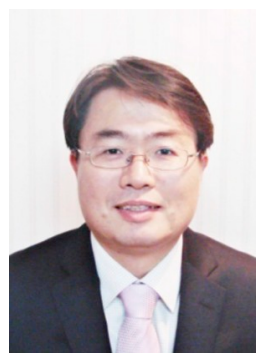

Joonho Bae received his B.S. and M.S. degrees from Seoul National University in 1996 and 1998, respectively. He was awarded the Ph.D. degree by the University of Texas, Austin, in 2007. He carried out postdoctoral research at Georgia Institute of Technology until 2011, and afterward worked in Samsung Electromechanics as a senior researcher. He joined the Department of Nano Physics in Gachon University in 2013. His recent research interests include the synthesis of nanomaterials for energy devices and quantum transport phenomena.

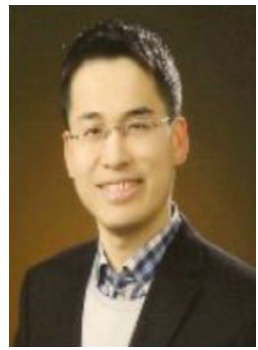

Dongseok Suh received his Ph.D. degree in Physics from Seoul National University in 2002. After working at the University of Texas, Dallas, and Samsung Electronics, he joined Sungkyunkwan University, South Korea, as a faculty in 2013. His current research interests include spinnable carbon nanotube sheets and yarns, two-dimensional materials and devices, and piezoelectricity. 